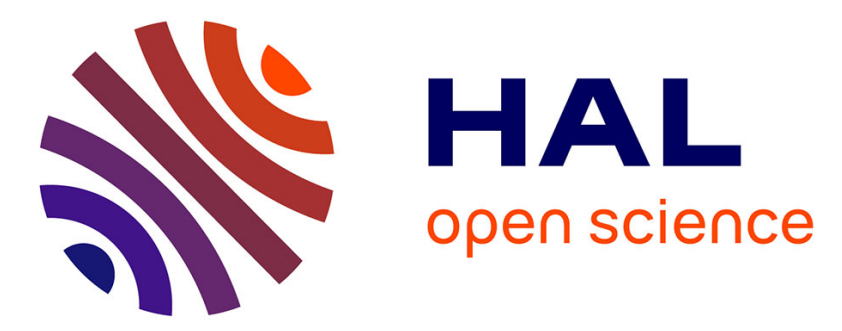

\title{
Success rate of a biological invasion in terms of the spatial distribution of the founding population
}

\author{
Jimmy Garnier, Lionel Roques, Francois Hamel
}

\section{To cite this version:}

Jimmy Garnier, Lionel Roques, Francois Hamel. Success rate of a biological invasion in terms of the spatial distribution of the founding population. Bulletin of Mathematical Biology, 2012, 74 (2), pp.453-473. 10.1007/s11538-011-9694-9 . hal-01257398

\section{HAL Id: hal-01257398 \\ https://hal.science/hal-01257398}

Submitted on 18 Jan 2016

HAL is a multi-disciplinary open access archive for the deposit and dissemination of scientific research documents, whether they are published or not. The documents may come from teaching and research institutions in France or abroad, or from public or private research centers.
L'archive ouverte pluridisciplinaire HAL, est destinée au dépôt et à la diffusion de documents scientifiques de niveau recherche, publiés ou non, émanant des établissements d'enseignement et de recherche français ou étrangers, des laboratoires publics ou privés. 


\title{
Success rate of a biological invasion in terms of the spatial distribution of the founding population
}

\author{
Jimmy Garnier ${ }^{a, b}$, Lionel Roques ${ }^{a, *}$ and François Hamel b,c \\ a UR 546 Biostatistique et Processus Spatiaux, INRA, F-84000 Avignon, France \\ b Aix-Marseille Université, LATP, Faculté des Sciences et Techniques \\ Avenue Escadrille Normandie-Niemen, F-13397 Marseille Cedex 20, France \\ c Institut Universitaire de France
}

June 27, 2011

\begin{abstract}
We analyze the role of the spatial distribution of the initial condition in reaction-diffusion models of biological invasion. Our study shows that in the presence of an Allee effect, the precise shape of the initial (or founding) population is of critical importance for successful invasion. Results are provided for one-dimensional and two-dimensional models. In the one-dimensional case, we consider initial conditions supported by two disjoint intervals of length $L / 2$ and separated by a distance $\alpha$. Analytical as well as numerical results indicate that the critical size $L^{*}(\alpha)$ of the population, where the invasion is successful if and only if $L>L^{*}(\alpha)$, is a continuous function of $\alpha$ and tends to increase with $\alpha$, at least when $\alpha$ is not too small. This result emphasizes the detrimental effect of fragmentation. In the two-dimensional case, we consider more general, stochastically generated initial conditions $u_{0}$, and we provide a new and rigorous definition of the rate of fragmentation of $u_{0}$. We then conduct a statistical analysis of the probability of successful invasion depending on the size of the support of $u_{0}$ and the fragmentation rate of $u_{0}$. Our results show that the outcome of an invasion is almost completely determined by these two parameters. Moreover, we observe that the minimum abundance required for successful invasion tends to increase in a nonlinear fashion with the fragmentation rate. This effect of fragmentation is enhanced as the strength of the Allee effect is increased.
\end{abstract}

Keywords: Reaction-diffusion; Allee effect; Biological invasions; Fragmentation; Initial condition.

${ }^{*}$ Corresponding author: lionel.roques@avignon.inra.fr. 


\section{Introduction}

Over the last decades, the number of biological invasions by alien organisms has increased exponentially (DAISIE, 2009), in large part because of human activities such as trade and travel. These biological invasions are characterized by a rapid expansion of the invasive organism's range. They are usually associated with biodiversity losses (IUCN, 2000, 2002; Kenis et al., 2009) and often cause significant harm to ecosystem functioning (Richardson et al., 2000; Vilà et al., 2009), socio-economic values (Kenis, 2006) and human health (Roques et al., 2009; Vilà et al., 2009) in the invaded regions.

The typical sequential stages of a successful invasion process begin with the introduction of a small founding population (that is, arrival), followed by the establishment and spread stages (Dobson and May, 1986; Walther et al., 2009). During the establishment stage, the individuals in the founding population reproduce and increase in number to form a colony that is self-perpetuating. The spread corresponds to the expansion of the species range into its new habitat.

Successful establishment often requires multiple introductions (Richardson et al., 2000; Walther et al., 2009). The success of the establishment phase indeed depends on the size of the founding population as well as on several endogenous and exogenous factors. Understanding the intertwined roles of these factors is of critical importance to slow down the rate at which biological invasions occur. In this paper, we investigate the interactions between founding population characteristics (namely, size and spatial distribution) and the profile of the growth function associated with the population.

Environmental factors such as resource availability, spatial heterogeneity (Berestycki et al., 2005; Cantrell and Cosner, 2003; Fahrig, 2003; Roques and Hamel, 2007; Roques and Stoica, 2007) and climate conditions (Roques et al., 2008; Walther et al., 2009) can play an important role in the persistence of the newly introduced population. Additionally, because of the typically small size of the founding population, it is widely accepted that the Allee effect plays a critical role in the success of establishment (Drake, 2004; Leung et al., 2004; Yamanaka and Liebhold, 2009). The Allee effect can be seen in many species (Allee, 1938; Dennis, 1989; Kramer et al., 2009; Veit and Lewis, 1996); it indicates that the per capita growth rate of individuals decreases at low densities. This effect may result from several processes that can co-occur (Berec et al., 2007), such as diminished chances of finding mates at low densities (Mccarthy, 1997), demographic stochasticity (Lande, 1998) or fitness decreases due to consanguinity.

In the present work, we analyze the effect of the spatial distribution of the founding population - that is, the repartition of the initial population into subgroups - on the success rate of an invasion. The relationships between successful establishment, the size of the founding population and the Allee effect have already been investigated in empirical (references above) and theoretical studies (Aronson and Weinberger, 1978; Du and Matano, 2010; Lewis and Kareiva, 1993; Murray, 2002; Turchin, 1998; Zlatoš, 2006). The influence of the corrugation of the edge of the founding population on the invasion speed has also been studied by Lewis and Kareiva (1993). However, the effect of the spatial distribution of the founding population has received less attention. Considering a closely related problem based on a stochastic reaction-diffusion equation with successive random immigration events, Drury et al. (2007) have shown the important role of the spatial 
distribution of the immigration events on the invasion risks. This suggests that the spatial distribution of the founding population, and in particular its rate of fragmentation, also plays an important role.

Because they draw on well-developed mathematical theory, reaction-diffusion models have been the main analytical framework to study the persistence and spread of biological organisms since the early work of Skellam (1951). One of the most frequent reaction-diffusion models is the Fisher-Kolmogorov-PetrovskyPiskunov (FKPP) (Fisher, 1937; Kolmogorov et al., 1937) equation, which was first intended to describe the propagation of advantageous genes inside a population. However, this equation does not take the Allee effect into account. Thus, in FKPP models, the persistence of the population does not depend at all on the characteristics of the initial population, even in heterogeneous environments (Berestycki et al., 2008, 2005, 2007). As a consequence, most studies that use FKPP-like models focus on the effects of environmental characteristics on persistence and spread (Berestycki et al., 2005; Cantrell and Cosner, 2003; Shigesada and Kawasaki, 1997) rather than on the effect of the initial condition. In contrast, we assume in this work that the underlying environment is homogeneous to isolate the effects of the characteristics of the initial population.

In the differential equations framework, the spatially homogeneous equation $N^{\prime}=f(N)$, with $f(N) \leq 0$ for $N$ below some threshold $\rho$, is a very simple example of a model involving an Allee effect. In this case, the dependence on the initial condition is obvious. In reaction-diffusion models, the Allee effect is typically modeled through bistable nonlinear terms (Fife, 1979; Turchin, 1998). The dependence of the behavior of such models on initial conditions was first investigated by Aronson and Weinberger (1978) and Fife and McLeod (1977), and then by Zlatoš (2006) and Du and Matano (2010) for one-dimensional models. In particular, for one-parameter families of initial conditions $u_{0}(L)$ that are strictly monotone with respect to the parameter $L$, it is shown in (Du and Matano, 2010) that there is a unique critical threshold $L=L^{*}$ between extinction and propagation (see Section 3 for more details).

In this paper, the effect of the spatial structure of the founding population $u_{0}$ (i.e., the initial condition in the model) is investigated for one-dimensional (1D) and two-dimensional (2D) models presented in Section 2.1. In both $1 \mathrm{D}$ and $2 \mathrm{D}$ cases, the initial conditions that we consider are binary functions which can only take the values 0 (absence of the species) and 1 (presence of the species). Because of their simple nature, these initial conditions are completely determined by their support, i.e., the regions where the species is present at $t=0$. This allows us to focus on the effect of the geometrical properties of the support, namely its size and its fragmentation rate, on the outcome of the invasion. In the $1 \mathrm{D}$ case, to derive analytical computations, we consider a simple class of initial conditions which include the initial conditions which have been considered by Zlatoš (2006). Their support is made of two intervals of the same length $L / 2$, separated by a distance $\alpha$, as described in Section 2.2.1. This distance is understood as a measure of the rate of fragmentation of $u_{0}$. In the $2 \mathrm{D}$ case, we consider more general, stochastically generated initial conditions, and we give a new rigorous definition of the rate of fragmentation in Section 2.2.2. Results are provided in Section 3. In the 1D case, the effects of fragmentation on the success of establishment and spread are investigated through analytical and numerical computations. In the $2 \mathrm{D}$ case, we carry out a statistical analysis of the probability of successful establishment, depending on the size of the support and 
the fragmentation rate of the initial condition.

\section{Materials and methods}

\subsection{The reaction-diffusion model}

Throughout this paper, we assume that the population density $u(t, x)$ is driven by the following reactiondiffusion equation:

$$
\begin{cases}u_{t}=D \Delta u+f(u), & \text { for } t>0 \text { and } x \in \mathbb{R}^{n} \\ u(0, x)=u_{0}(x), & \text { for } x \in \mathbb{R}^{n},\end{cases}
$$

with $n=1$ or $n=2$ and $D>0$.

The non-linear growth term $f(u)$ in $(2.1)$ is assumed to satisfy:

$$
f \in C^{1}(\mathbb{R}), f^{\prime} \text { is Lipschitz-continuous, } f(0)=f(1)=0 \text { and } \int_{0}^{1} f(s) d s>0 .
$$

In addition, we assume that the function $f$ takes a strong Allee effect into account:

$$
\text { There exists } \rho \in(0,1) \text { such that } f<0 \text { in }(0, \rho) \text { and } f>0 \text { in }(\rho, 1) \text {. }
$$

This hypothesis means that the growth rate $f(u)$ is negative at low densities, which corresponds to a strong Allee effect. The parameter $\rho$ corresponds to the so-called "Allee threshold", below which the growth rate becomes negative. It therefore measures the strength of the Allee effect. Under these assumptions, the solution to (2.1) may have several behaviors, depending on $f$ and the initial density at $t=0$. To provide a biological interpretation of these possible behaviors, we make the following definitions:

Definition 1 (Founding population). The founding population corresponds to the initial population at $t=0: u_{0}(x)=u(0, x) \in[0,1]$ for $x \in \mathbb{R}^{n}$, which is assumed to be compactly supported.

Definition 2 (Successful establishment). We say that the establishment is successful if $u(t, x)$ converges to a positive stationary state as $t \rightarrow \infty$.

Definition 3 (Successful invasion). We say that the invasion is successful if $u(t, x)$ converges to the stationary state 1 as $t \rightarrow \infty$.

Definition 4 (Establishment failure). We say that the establishment phase has failed if $u(t, x)$ converges to the stationary state 0 uniformly in $x$ as $t \rightarrow \infty$.

Remark 1. Note that successful establishment without successful invasion is possible but rare (see Section 3.1). In the $1 D$ case, when the invasion is successful in the sense of Def. 3, then

$$
\begin{cases}\min _{|x| \leq c t} u(t, x) \rightarrow 1 \text { as } t \rightarrow \infty & \text { for each } 0 \leq c<c_{0}, \\ \max _{|x| \geq c^{\prime} t} u(t, x) \rightarrow 0 \text { as } t \rightarrow \infty & \text { for each } c^{\prime}>c_{0} .\end{cases}
$$


Note that $c_{0}>0$ is the unique speed of the traveling front $\phi\left(x-c_{0} t\right)$ connecting 0 to 1 at $+\infty$ and $-\infty$ respectively (Aronson and Weinberger, 1978).

For real populations, successful establishment without invasion can occur (several examples are given in DAISIE, 2009, 2010). This corresponds to steady state populations with finite range. The existence of such steady states can be a consequence of environmental heterogeneity (e.g. the location of the species bioclimatic envelop, see Section 3.2 in Hamel et al., 2010). In such heterogeneous environments, even when the population can adapt to its local conditions, gene flow can cause maladaptation of the peripheral groups and lead to finite range populations (Kirkpatrick and Barton, 1997).

\subsection{Initial conditions}

Depending on the founding population $u_{0}$, establishment failure or establishment success can occur. To study this dependence, we consider particular types of initial binary conditions, namely $u_{0}=1$ or 0 almost everywhere. Note that support may vary in size and shape; we focus in particular on the fragmentation of this support.

\subsubsection{One-dimensional case}

To derive analytical computations in the case $n=1$, we consider a simple case of fragmentation.

$$
u_{0}(x)=\chi_{[-(\alpha / 2+L / 2),-\alpha / 2]}(x)+\chi_{[\alpha / 2, \alpha / 2+L / 2]}(x) \text { for } x \in \mathbb{R} .
$$

Note that for any set $J \subset \mathbb{R}, \chi_{J}$ is the characteristic function of $J$, that is, $\chi_{J}(x)=1$ if $x \in J$ and $\chi_{J}(x)=0$ otherwise.

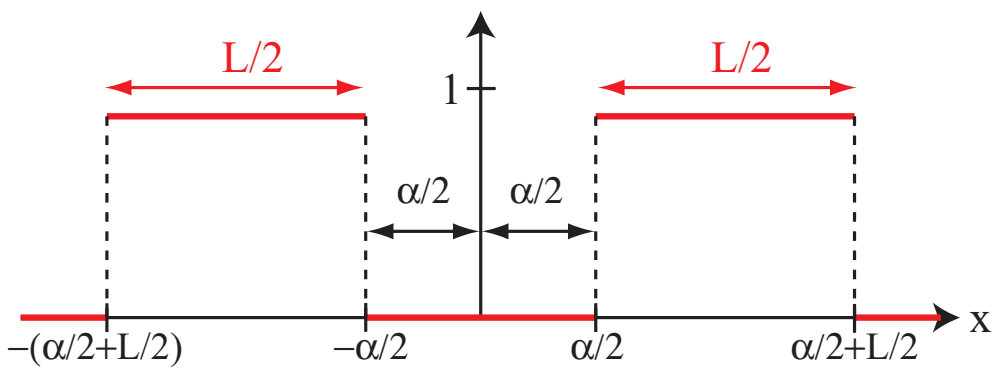

Figure 1: An initial condition $u_{0}$ as defined by (2.4).

This corresponds to an initial population density, whose support is split into two equal parts of length $L / 2>0$ separated by a distance $\alpha$ (see Fig. 1). Problem (2.1) with "aggregated" initial conditions (i.e., with $u_{0}=\chi_{[-L / 2, L / 2]}$, that is, $\alpha=0$ ) has been investigated by several authors (Kanel, 1964; Zlatoš, 2006). 
Remark 2. The problem (2.1), with $n=1$ and initial condition (2.4), is equivalent to the Neumann problem.

$$
\left\{\begin{array}{l}
u_{t}=D u_{x x}+f(u) \text { for } t>0 \text { and } x \geq 0 \\
u_{x}(t, 0)=0 \text { for } t>0 \\
u(0, x)=\chi_{(\alpha / 2, \alpha / 2+L / 2]}(x) \text { for } x \geq 0
\end{array}\right.
$$

Indeed, if $u$ solves (2.1), (2.4), then $u(t,-x)$ also solves (2.1), (2.4). By uniqueness, we obtain that $u(t, x)=u(t,-x)$ for all $t>0$ and $x \in \mathbb{R}$. Thus, $u$ also satisfies (2.5). Conversely, by uniqueness of the solution of (2.5) and by extending it by symmetry on $(-\infty, 0]$, we obtain equivalence between (2.1), (2.4) and (2.5).

\subsubsection{The two-dimensional case, the notion of abundance and the measure of fragmentation}

The effect of the spatial distribution of the founding population can also be assessed in the two-dimensional case $n=2$ through the numerical investigation of the behavior of the model solution (2.1) over a large number of stochastically generated initial conditions.

As in the $1 \mathrm{D}$ case, we assume the initial conditions $u_{0}$ to be binary functions, where

$$
\text { at each point }(x, y) \text { in } \mathbb{R}^{2}, u_{0}(x, y) \text { either takes the value } 0 \text { or } 1 \text {. }
$$

We furthermore assume that the support of $u_{0}$ is included in a closed square $C$ :

$$
\text { for all }(x, y) \in \mathbb{R}^{2}, u(x, y)=0 \text { if }(x, y) \notin C \text {. }
$$

To build samples of functions $u_{0}$ with various spatial distributions, we used a model developed by Roques and Stoica (2007) and inspired by statistical physics. In this model, the square $C$ is divided into $n_{C}$ closed equi-measurable subsquares $C_{i}$, with interiors being pairwise disjoint. The obtained functions $u_{0}$ satisfy:

$$
\text { for all } i=1, \ldots, n_{C}, u_{0} \text { is constant over the interior of } C_{i} \text {. }
$$

As key properties, this stochastic model allows exact control over the population abundance level

$$
p\left(u_{0}\right)=\frac{1}{|C|} \int_{C} u_{0}(x, y) d x d y,
$$

and leads to configurations with different levels of fragmentation. The notion of fragmentation is defined as follows.

The lattice made of cells $C_{i}$ is equipped with a four-neighborhood system $V\left(C_{i}\right)$ (Fig. 2). For each function $u_{0}$, constant on the subsquares $C_{i}$, we define

$$
s\left(u_{0}\right)=\frac{1}{2} \sum_{C_{i} \subset C} \sum_{C_{j} \in V\left(C_{i}\right)} \mathbb{1}\left\{u_{0}\left(C_{j}\right)=u_{0}\left(C_{i}\right)=1\right\},
$$




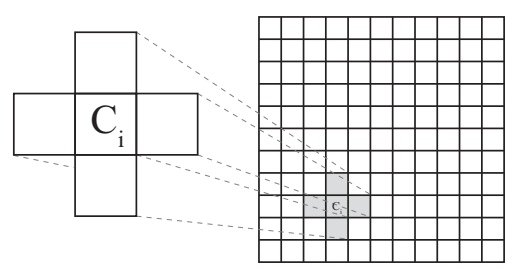

Figure 2: The four-neighborhood system: an element $C_{i}$ of $C$ and its four neighbors.

the number of pairs of neighbors $\left(C_{i}, C_{j}\right)$ such that $u_{0}$ takes the value 1 on $C_{i}$ and $C_{j}$. Then $\mathbb{1}\{\cdot\}$ denotes the indicator function, namely, $\mathbb{1}\{P\}=1$ if property $P$ is satisfied and 0 , otherwise. As already observed in the literature (Roques and Chekroun, 2007, 2010; Roques and Stoica, 2007), the value $s\left(u_{0}\right)$ is directly linked to fragmentation. Assuming abundance $p\left(u_{0}\right)$ is fixed, the higher $s\left(u_{0}\right)$ is, the more aggregated the region $\left\{u_{0}=1\right\}$ is.

We define pattern fragmentation by comparing the value of $s\left(u_{0}\right)$ with the maximum possible value of the index $s$ among functions with a given abundance $p$. This maximum value $B[p]$ can be computed explicitly as an immediate consequence of the results of Harary and Harborth (1976) on polyominoes.

Lemma 1 (Harary and Harborth (1976)). Choose $p \in[0,1]$ such that $p \times n_{C} \in \mathbb{N}$ and

$$
U_{0}:=\left\{u_{0} \text { satisfying }(2.6)-(2.8) \text {, and } p\left(u_{0}\right)=p\right\} \text {. }
$$

We have

$$
B[p]:=\max _{u_{0} \in U_{0}} s\left(u_{0}\right)=2 n_{+}-\left\lceil 2 \sqrt{n_{+}}\right\rceil,
$$

where $n_{+}=p \times n_{C}$ and $\left\lceil 2 \sqrt{n_{+}}\right\rceil$is the smallest integer that is greater than or equal to $2 \sqrt{n_{+}}$.

Then, we set

$$
f r\left(u_{0}\right)=1-\frac{s\left(u_{0}\right)}{B\left[p\left(u_{0}\right)\right]} .
$$

The index $f r$ belongs to $[0,1]$. Whatever the abundance $p$ is, the most aggregated configurations (in the sense $s\left(u_{0}\right)=B[p]$ ) verify $f r\left(u_{0}\right)=0$, whereas the most fragmented configurations (in the sense $s\left(u_{0}\right)=0$ ) verify $f r\left(u_{0}\right)=1$. In the sequel, $f r\left(u_{0}\right)$ is referred to as the fragmentation rate of $u_{0}$. Samples of functions $u_{0}$ obtained with the model from (Roques and Stoica, 2007) with various values of $f r\left(u_{0}\right)$ and $p\left(u_{0}\right)$ are shown in Fig. 3.

Remark 3. There exist several ways of generating binary patterns. The most common are neutral models, originally introduced by Gardner et al. (1987). They can include parameters that regulate fragmentation (Keitt, 2000). The model that we use here is also a neutral model in the sense that it is a stochastic model of pattern generation. The abundance $p$ and the number of pairs $s$ are controlled during the process of pattern generation; this allows for good sampling over the parameter space $(f r, p) \in[0,1] \times[0,1]($ see Fig. 5). 


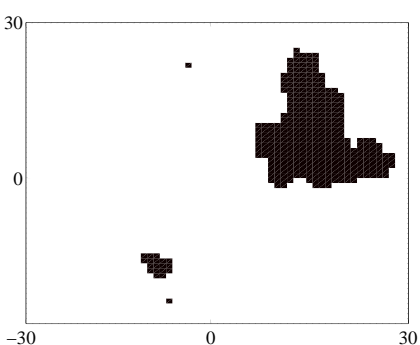

(a) $p=0.1, f r=0.05$

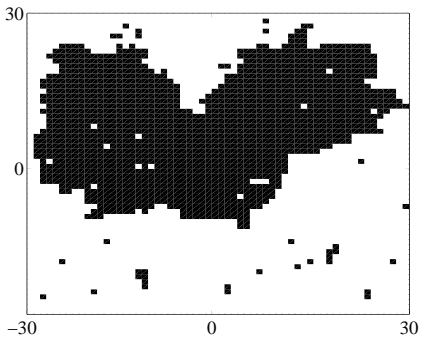

(d) $p=0.4, f r=0.05$

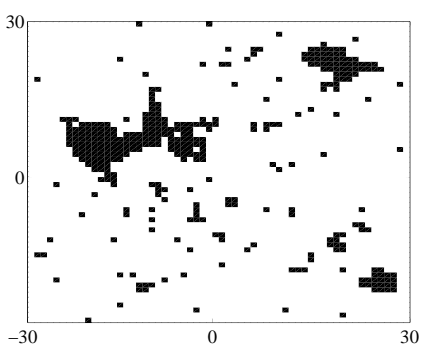

(b) $p=0.1, f r=0.4$

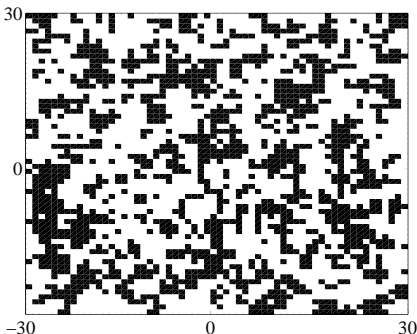

(e) $p=0.4, f r=0.4$

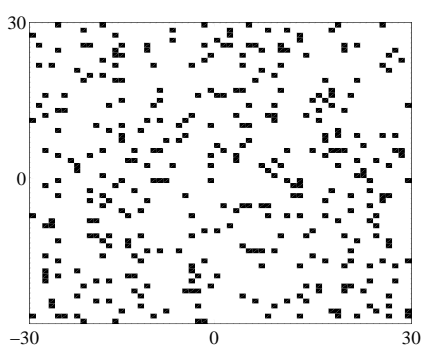

(c) $p=0.1, f r=0.9$

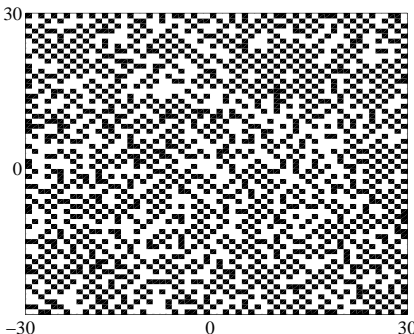

(f) $p=0.4, f r=0.9$

Figure 3: Initial conditions $u_{0}$ in the two-dimensional case: examples of patterns with different abundance and fragmentation rates. Note that $C=[-30,30] \times[-30,30]$ and $n_{C}=3600$. 


\section{Results}

In this section, we present analytical and numerical results on the effect of the spatial distribution of the founding population $u_{0}$ on the outcome of the invasion modeled by equation (2.4).

\subsection{Analytical result in the one-dimensional case}

We first mention some results in (Du and Matano, 2010) that prove the existence of a threshold value for the size of the support of $u_{0}$ such that establishment fails (i.e., $u(t, x) \rightarrow 0$ as $t \rightarrow \infty$ ) if and only if the size of the support is below this threshold, and invasion is successful (i.e., $u(t, x) \rightarrow 1$ as $t \rightarrow \infty$ ) if and only if the size of the support is above this threshold. The symmetry of the problem $(2.1),(2.4)$ together with the uniqueness of its solution imply that the solution is symmetrical with respect to $\{x=0\}$ for all $t \geq 0$.

Theorem 1 (case $\alpha=0$ : Zlatoš (2006), case $\alpha \geq 0$ : Du and Matano (2010)). Let $\alpha \geq 0$. Assume that $f$ satisfies (2.2-2.3). Then, there exists $L^{*}(\alpha)>0$ such that:

i For any $L<L^{*}(\alpha)$, the solution to (2.1), (2.4) converges to 0 as $t \rightarrow \infty$, uniformly in $\mathbb{R}$.

ii For any $L>L^{*}(\alpha)$, the solution to (2.1), (2.4) converges to 1 as $t \rightarrow \infty$, uniformly on compacts.

iii If $L=L^{*}(\alpha)$, the solution to (2.1), (2.4) converges to a positive stationary solution $\tilde{u}$ uniformly in $\mathbb{R}$ as $t \rightarrow+\infty$, where $\tilde{u}$ verifies $-D \tilde{u}^{\prime \prime}=f(\tilde{u}), \tilde{u}(0)=\rho_{1}:=\sup \left\{\rho^{\prime} \in(0,1)\right.$ such that $\left.\int_{0}^{\rho^{\prime}} f(s) d s \leq 0\right\}$ and $\tilde{u}^{\prime}(0)=0$. Moreover, $\tilde{u}$ is symmetrically decreasing, i.e., $\tilde{u}(x)=\tilde{u}(-x)$ and $\tilde{u}^{\prime}(x)<0$ for all $x>0$.

The next result analyzes the effect of fragmentation by studying the dependence of the threshold value $L^{*}(\alpha)$ with respect to the size $(\alpha)$ of the gap between the two components of the support of $u_{0}$.

Theorem 2. Let $\alpha \geq 0$ and $L^{*}(\alpha)$ be defined by Theorem 1. Then the function $\alpha \mapsto L^{*}(\alpha)$ is continuous on $[0,+\infty)$. Furthermore, $L^{*}(\alpha)<2 L^{*}(0)$ for all $\alpha \geq 0$ and

$$
L^{*}(\alpha) \rightarrow 2 L^{*}(0) \text { as } \alpha \rightarrow \infty .
$$

This result highlights the global detrimental effect of fragmentation on the success of establishment and invasion. Indeed, when the gap $\alpha$ between the two components of the support of $u_{0}$ is large, the minimal viable size $L^{*}(\alpha)$ of support of the founding population tends to become twice as large as if the individuals were gathered in a single group. However, the strict sign in the inequality $L^{*}(\alpha)<2 L^{*}(0)$ shows that, whatever the distance $\alpha$ separating the two groups, there is at least some cooperation between them. Moreover, the result of Theorem 2 implies that the amplitude of variation of the threshold $L^{*}(\alpha)$ is at least equal to $L^{*}(0)$. Thus, the spatial structure of the support of the founding population $u_{0}$ plays an important role on invasion success. The proof of Theorem 2 is presented in Appendix A.

In the next section, we use numerical computations to study whether there is an increasing relationship between $L^{*}(\alpha)$ and the distance $\alpha$. 


\subsection{Numerical results}

We carried out numerical computations in one- and two-dimensional space. We refer to Appendix B for some details on the numerical method that was used for solving equation (2.1). In these numerical computations, the function $f$ in (2.1) was assumed to be of the following cubical form:

$$
f(s)=r s\left(1-\frac{s}{K}\right)\left(\frac{s-\rho}{K}\right),
$$

with $\rho \in(0, K / 2)$. Such a growth function is a typical example of a reaction term involving an Allee effect (Keitt et al., 2001; Lewis and Kareiva, 1993; Roques et al., 2008). Without loss of generality, with the substitutions $v(t, x)=u(t, x) / K$ and $w(t, x)=v(t / r, x \sqrt{D / r})$ into equation (2.1), we can assume that $K=r=D=1$ and $\rho \in(0,1 / 2)$. Thus,

$$
f(s)=s(1-s)(s-\rho)
$$

Since $\rho \in(0,1 / 2)$, the quantity $\int_{0}^{1} f(s) d s$ is positive. The function $f$ defined by $(3.11)$ therefore fulfils the assumptions $(2.2-2.3)$.

\subsubsection{The one-dimensional case}

The aim of this section is (i) to study the relationship between the minimum viable population size $L^{*}(\alpha)$ and the distance $\alpha$ and (ii) to study the effect of the Allee threshold $\rho$ on the above relationship. The value of $L^{*}(\alpha)$, as defined in Theorem 1 , has been computed for $\alpha \in[0,16]$ and several values of the parameter $\rho$.

The results of these computations are depicted in Fig. 4. As expected from Theorem $2, L^{*}(\alpha) \rightarrow 2 L^{*}(0)$ for large values of $\alpha$ : for each value of $\rho, L^{*}(\alpha)$ is very close to $2 L^{*}(0)$ when $\alpha=16$. This justifies the choice of the study interval $\alpha \in[0,16]$. Moreover, Fig. 4 shows an increasing trend between the threshold $L^{*}(\alpha)$ and $\alpha$, at least when $\alpha$ is not too small. This increasing relationship means that the more fragmented the founding population is, the larger it needs to be for the invasion to be successful. Additionally, the dependence between $L^{*}(\alpha)$ and $\alpha$ is nonlinear; the curves corresponding to $L^{*}(\alpha)$ have a steeper slope for intermediate values of $\alpha$.

However, we observe that for small values of $\alpha, L^{*}(\alpha)$ slightly decreases with $\alpha$. This phenomenon can be clearly seen on Fig. 4 for $\rho \geq 0.4$; the numerical simulations indicate that it is also true for smaller values of $\rho$. In particular, $L^{*}(\alpha)$ can reach values smaller than $L^{*}(0)$. Roughly speaking, this means that when $\alpha$ is very small, the gap between $-\alpha / 2$ and $\alpha / 2$ is filled by diffusion at a time (of the order $\alpha^{2}$ ) which is much smaller than the time it takes for the interfaces at the positions $-(\alpha / 2+L / 2)$ and $(\alpha / 2+L / 2)$ to move.

Regarding the effect of $\rho$, we observe that $L^{*}(\alpha)$ tends to increase with $\rho$. This is an obvious consequence of the parabolic maximum principle; for any $\alpha \geq 0, L>0$ and $\rho \in(0,1 / 2)$, let us denote the solution to (2.1), (2.4) by $u_{\rho}^{L, \alpha}(t, x)$, where $f$ is defined by (3.11). We thus have the following lemma: 




Figure 4: Value of the critical threshold $L^{*}(\alpha)$ in terms of the distance $\alpha$ and the Allee threshold $\rho$. For each value of $\rho$, the dotted line corresponds to the associated value of $2 L^{*}(0)$.

Lemma 2. Let $0<\rho_{1} \leq \rho_{2}<1 / 2$. For all $\alpha \geq 0$ and $L>0$,

$$
u_{\rho_{1}}^{L, \alpha}(t, x) \geq u_{\rho_{2}}^{L, \alpha}(t, x) \text { for all } t \geq 0, x \in \mathbb{R} .
$$

The proof of this lemma is given in Appendix A. According to the definition of $L^{*}(\alpha), L^{*}(\alpha)$ is an increasing function of $\rho$. In particular, $L^{*}(0)$ is an increasing function of $\rho$. Thus, the result of Theorem 2 together with the monotonic trend between $\alpha$ and $L^{*}(\alpha)$ imply that the amplitude of the variation of the critical size $L^{*}(\alpha)$ (that is, the quantity $\left.\sup _{\alpha} L^{*}(\alpha)-\inf _{\alpha} L^{*}(\alpha)\right)$ tends to increase as $\rho$ increases. In other words, the effect of fragmentation tends to increase with the strength of the Allee effect.

\subsubsection{The two-dimensional case}

The aim of this section is to study the effect of spatial fragmentation of the founding population's support in more realistic scenarios than those studied in the $1 \mathrm{D}$ case.

The model (2.1), with the function $f$ defined by (3.11), was solved using stochastically generated initial conditions $u_{0}$ that satisfy the conditions (2.6)-(2.8), as described in Section 2.2.2. Those conditions $u_{0}$ are characterized by two indices, namely, an abundance index $p\left(u_{0}\right) \in[0,1]$ and a fragmentation rate $\operatorname{fr}\left(u_{0}\right) \in[0,1]$. For our computations, we assumed that the support of the initial conditions was included 
in the set $C=[-30,30] \times[-30,30]$, which was divided into $n_{C}=60 \times 60$ subsquares $C_{i}$, on which the functions $u_{0}$ are constant.

Using the stochastic model described in Section 2.2.2, we derived $1.5 \cdot 10^{4}$ functions $u_{0, k}, k=1 \ldots 1.5 \cdot 10^{4}$, with various abundance indices and fragmentation rates. Then, (2.1) was solved with each $u_{0}$ as initial condition. The computations were carried out with several values of the Allee threshold, including $\rho=$ $0.1,0.3$ and 0.45 . Some details on the numerical method which was used to solve this equation are given in Appendix B.

The results of these computations are presented in Fig. 5. Each point in the figure is attached to a certain behavior of the model, namely, successful establishment or establishment failure. It appears that for each value of $\rho$, there are two distinct regions in the parameter space $(f r, p)$, one corresponding to successful establishment and the other one to establishment failure. These regions are separated by a narrow interface in which the two behaviors are possible. In this narrow interface, and in the surrounding regions of the parameter space, the proportion of successful establishment cannot be directly inferred from Fig. 5. This proportion corresponds to the probability of successful establishment.

Remark 4. In the region of the parameter space $(f r, p)$ where $p>0.5$, establishment was always successful. Thus, this region is not represented in Fig. 5.

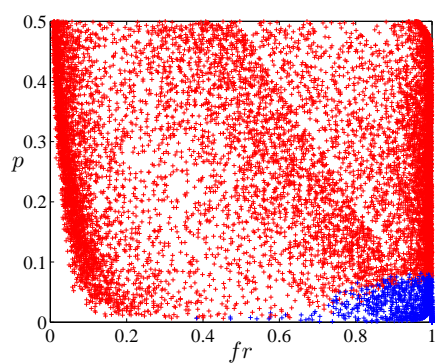

(a) $\rho=0.1$

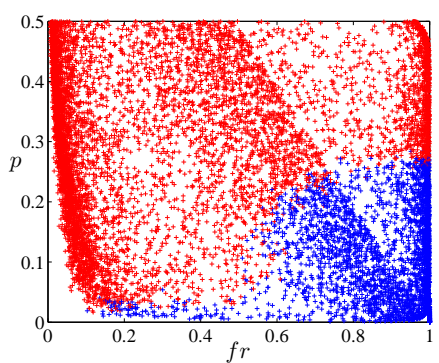

(b) $\rho=0.3$

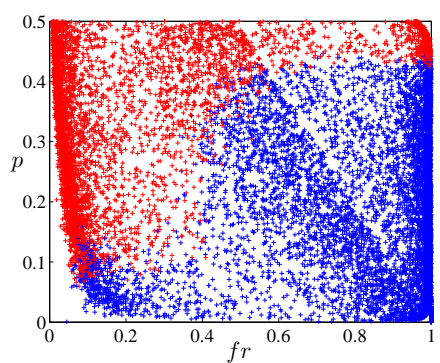

(c) $\rho=0.45$

Figure 5: Establishment success for the initial conditions $u_{0, k}, k=1 \ldots 1.5 \cdot 10^{4}$, in terms of the fragmentation rate $\operatorname{fr}\left(u_{0, k}\right)$ and abundance $p\left(u_{0, k}\right)$, for three values of the Allee threshold $\rho$. Red crosses correspond to successful establishment and blue crosses correspond to establishment failure.

To study the probability of successful establishment in terms of the values of $f r$ and $p$, we defined a function $Y$ such that $Y\left(u_{0, k}\right)=1$ if the initial condition $u_{0, k}$ leads to successful establishment and $Y\left(u_{0, k}\right)=0$, otherwise. We then applied a smoothing regression to the data $\left(f r\left(u_{0, k}\right), p\left(u_{0, k}\right), Y\left(u_{0, k}\right)\right)$. More precisely, the data were fitted with an algorithm to replace each point with the average of the closest points surrounding it (i.e., in a rectangular neighborhood of size $0.1 \times 0.05$ ). That is, $Y$ was fitted by the 
estimator $\hat{Y}$, which is defined over $[0,1] \times[0,1]$ :

$$
\hat{Y}(f r, p)=\frac{\sum_{\substack{\left(f r\left(u_{0, k}\right), p\left(u_{0, k}\right)\right)-(f r, p) \|<\varepsilon \\ k=1 \ldots 1.5 \cdot 10^{4}}} Y\left(f r\left(u_{0, k}\right), p\left(u_{0, k}\right)\right)}{\sum_{\substack{\left.\| f r\left(u_{0, k}\right), p\left(u_{0, k}\right)\right)-(f r, p) \|<\varepsilon \\ k=1 \ldots 1.5 \cdot 10^{4}}} 1} .
$$

Thus, to each point $(f r, p)$ in $[0,1] \times[0,1]$ is attached a value of $\hat{Y}(f r, p)$ which measures the probability of successful establishment. This function $\hat{Y}(f r, p)$ is depicted in Fig. 6 , for $\rho=0.1,0.3$, and 0.45 .

An analysis of Fig. 6 reveals that for each fixed value of $p$, the probability of successful establishment decreases with the fragmentation rate $f r$. Moreover, the interface in which the probability $\hat{Y}(f r, p)$ is neither close to 0 nor to 1 is narrow in the parameter space (that is, the region where $\hat{Y}(f r, p) \in(0.1,0.9)$ occupies approximately $1 / 40,1 / 16,1 / 12$ of the parameter space $[0,1] \times[0,1]$ for $\rho=0.1,0.3$ and 0.45 , respectively). Thus, the behavior of the solution of (2.1) with an initial condition $u_{0}$ satisfying (2.6)-(2.8) is almost completely determined by $f r$ and $p$.

For each $f r \in[0,1]$, let us define $P_{1 / 2}(f r)$ as the minimum abundance such that establishment is successful with probability $1 / 2$. The yellow curves in Fig. 6 correspond to a probability $1 / 2$ of successful establishment). We observe that for each value of the Allee threshold $\rho, P_{1 / 2}(1)$ is much larger than $P_{1 / 2}(0)$. Thus, the minimum required abundance for the establishment to be successful is much larger for fragmented founding populations as compared to aggregated ones. Furthermore, the relationship $f r \mapsto$ $P_{1 / 2}(f r)$ is comparable to the dependence $\alpha \mapsto L^{*}(\alpha)$, which was observed in the 1D case (Section 3.2.1). This dependence is highly nonlinear. On the one hand, for aggregated initial conditions (i.e., with small values of $\left.f r\left(u_{0}\right)\right)$ as well as very fragmented initial conditions (i.e., with values of $f r\left(u_{0}\right)$ close to 1 ), this dependence is almost flat. On the other hand, for intermediate values of the fragmentation rate, we observe a sudden increase in $P_{1 / 2}(f r)$, especially when $\rho=0.3$ and $\rho=0.45$. For $\rho=0.3$, we have $P_{1 / 2}(0.6)-P_{1 / 2}(0.52)=0.15$, and for $\rho=0.45$, we have $P_{1 / 2}(0.5)-P_{1 / 2}(0.36)=0.23$. Note that the probability of successful establishment decreases with $\rho$, especially for a fragmented initial condition. This property is the counterpart in the two-dimensional case of the monotonicity of $L^{*}(\alpha)$ with respect to $\rho$ mentioned in Section 3.2.1 (see Lemma 2).

Remark 5. - We note in Fig. 6 that the minimum abundance $P_{1}(f r)$ such that establishment probability

$\hat{Y}\left(f r, P_{1}(f r)\right)$ is equal to 1 converges to a value close to $\rho$ in the three cases considered here as $f r \rightarrow 1$. This is the case for $\rho=0.1: P_{1}(1)=0.11$, for $\rho=0.3: P_{1}(1)=0.31$ and for $\rho=0.45$ : $P_{1}(1)=0.47$. An explanation of this fact is as follows. Let $\tilde{u}$ be the solution of the following problem.

$$
\left\{\begin{array}{l}
\tilde{u}_{t}=\Delta \tilde{u}+f(\tilde{u}) \text { for } t>0, x \in C, \\
\tilde{u} \text { satisfies Neumann conditions on the boundary of } C \text { for } t>0, \\
u(0, x)=u_{0}(x) \text { for } x \in C
\end{array}\right.
$$




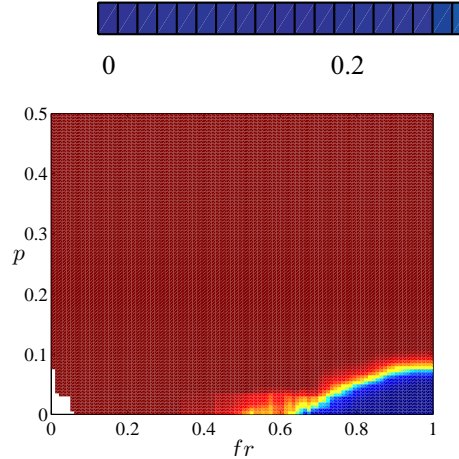

(a) $\rho=0.1$

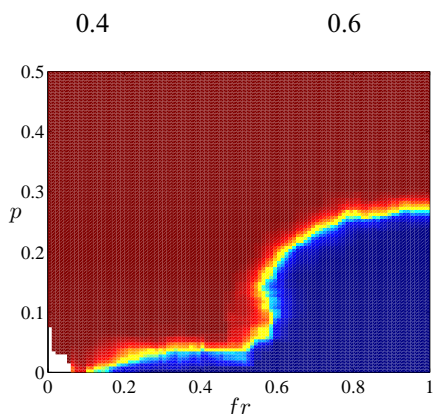

(b) $\rho=0.3$
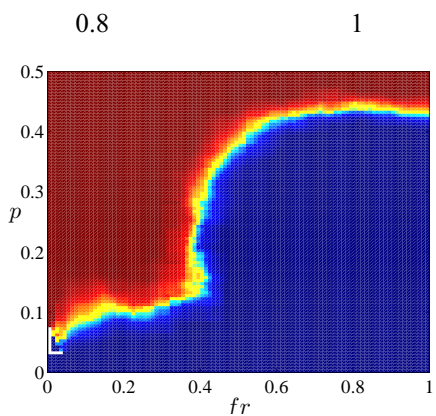

(c) $\rho=0.45$

Figure 6: Probability of successful establishment measured through $\hat{Y}$, in terms of the fragmentation rate $f r$ and the abundance $p$ of the founding population.

with $f$ satisfying (2.2) and (2.3) and $u_{0}$ satisfying (2.6), (2.8) and $f r\left(u_{0}\right)=1$. We conjecture that as the number $n_{C}$ of subsquares $C_{i}$ converges to $+\infty$, the behavior of $\tilde{u}$ (i.e., convergence to 0 or to 1 as $t \rightarrow \infty$ ) becomes similar to the behavior of a homogenized problem in which $u_{0}$ is replaced with the constant initial condition $\overline{u_{0}}=\frac{1}{|C|} \int_{C} u_{0}(s) d s=p\left(u_{0}\right)$, which in turn is equivalent to the ordinary differential equation $N^{\prime}=f(N)$ with initial condition $N(0)=p\left(u_{0}\right)$. Thus, in this asymptotic regime, we would obtain $\tilde{u}(t, x) \rightarrow 1$ in $C$ if and only if $p\left(u_{0}\right)>\rho$. The comparison principle implies that if the problems (2.1) and (3.13) are solved with the same initial condition $u_{0}$, we have $u \leq \tilde{u}$ for $t>0$ and $x \in C$. As a consequence, we finally obtain the inequality $P_{1}(1) \geq \rho$, for adequately large $n_{C}$.

- Using a formula by Lewis and Kareiva (1993) we can compute the minimum abundance for successful establishment when the initial condition is ball-shaped:

$$
p_{B}=\frac{\pi}{2|C|} \frac{1}{(1 / 2-\rho)^{2}} .
$$

For $\rho=0.1$, we get $p_{B}=310^{-3}$, for $\rho=0.3$, we have $p_{B}=0.01$ and for $\rho=0.45$, we obtain $p_{B}=0.17$. We can observe in Fig. 6 that these values of $p_{B}$ are not too far from the values of the minimum abundances $P_{1}(f r)$ for small values of $f r$. This is not surprising when $f r$ is close to 0 since the initial condition consists in a single aggregated group (Harary and Harborth, 1976).

\section{Discussion}

We have analyzed the role of the spatial distribution of the initial condition in reaction-diffusion models of biological invasions. These models incorporate reaction terms that take the Allee effect into account. 
In many simplified models, the Allee effect is not taken into account. The initial conditions therefore do not play an important role. This is probably why a number of recent studies have focused on the effect of environmental heterogeneities rather than on the role of initial conditions. However, the Allee effect is known to occur in many invasive species. In such cases, the precise shape of the initial (or founding) population is of crucial importance when predicting successful invasion, as emphasized in our study.

Our first result is an analytical result. In the 1D case, we considered schematic examples of founding populations that were made of two identical groups separated by a distance $\alpha$.

We have shown that the minimum size $L^{*}(\alpha)$ of the support of the founding population for successful establishment and invasion is a continuous function of the distance $\alpha$. Moreover, when this distance becomes large, $L^{*}(\alpha)$ becomes twice as large as if the individuals were assembled in a single group. This result shows that the spatial structure of the founding population indeed has an effect on the success of an invasion. Moreover, it is a first indication of the adverse effect of fragmentation. This adverse effect is confirmed by our numerical simulations. As illustrated in Fig. 4, the minimum viable size $L^{*}(\alpha)$ tends to increase with the distance $\alpha$ that separates the two subgroups, at least for not too small values of $\alpha$. The amplitude of variation of the minimum viable size with respect to $\alpha$ (that is, the quantity $\sup _{\alpha} L^{*}(\alpha)-\inf _{\alpha} L^{*}(\alpha)$ ) also tends to increase with the strength of the Allee effect as measured by the Allee threshold $\rho$. It should nevertheless be noted that for small values of $\alpha$, the minimum viable size decreases slightly with $\alpha$. Thus, the breaking apart of the founding population into two subgroups may have a beneficial effect on the success of the invasion when these subgroups are very close to each other. From a mathematical standpoint, the proof of the non-monotonicity of the function $\alpha \mapsto L^{*}(\alpha)$ is a challenging open problem.

In the $2 \mathrm{D}$ case, we considered much more realistic examples of founding populations. Using a stochastic model of pattern generation, we constructed a large number of initial conditions with various abundances (measured by the index $p \in[0,1]$ ) and fragmentation rates. Using a new, rigorous definition of the fragmentation rate (measured by the index $f r \in[0,1]$; see eq. (2.9)), we were able to describe the outcome of an invasion in terms of two attributes of the founding population $u_{0}$, namely, $p\left(u_{0}\right)$ and $f r\left(u_{0}\right)$. Interestingly, the outcome of the invasion is indeed almost completely determined by the values of $p\left(u_{0}\right)$ and $f r\left(u_{0}\right)$; for each value of the Allee threshold $\rho$, we obtained two distinct regions in the parameter space $(f r, p)$. One region corresponds to successful establishment, and the other one corresponds to establishment failure. The interface between these regions corresponds to a narrow region in the parameter space (Figs. 5 and 6 ). In our computations, we also observed that the set of initial conditions leading to successful establishment without successful invasion was very small (see also Remark 1 and Theorem 1 in the $1 \mathrm{D}$ case).

The results of Section 3.2.2 also show that the minimum abundance $p$ required for successful establishment tends to increase with the fragmentation rate $f r$. As in the $1 \mathrm{D}$ case, the effect of fragmentation is enhanced as the Allee threshold $\rho$ is increased. In addition, the relationship between the minimum abundance and the fragmentation rate is highly nonlinear and steep, threshold-like. Indeed, there is threshold value, say $f r^{*}$, where the minimum abundance for successful establishment dramatically increases. On both sides of this threshold, establishment success is almost independent on $f r$. On the one hand, if the 
rate of fragmentation is higher than $f r^{*}$, then the behavior of the model is close to the behavior of a homogenized problem (see first point in Remark 5). On the other hand, if the rate of fragmentation is lower than $f r^{*}$, the model almost behaves as if the initial condition was replaced by a single ball-shaped group (see second point in Remark 5). The existence of such a threshold $f r^{*}$ implies that small changes in the fragmentation rate can therefore drastically modify the outcome of an invasion if $f r$ is close to $f r^{*}$.

Finally, we have shown that the fragmentation of the support of a founding population $u_{0}$ tends to have a negative effect on invasion success in a homogeneous environment. We might wonder what would happen in heterogeneous environments. That is, instead of (2.1), consider the following model

$$
\left\{\begin{array}{l}
u_{t}=D \Delta u+f(u)+\nu(x) u, \text { for } t>0 \text { and } x \in \mathbb{R}^{n}, \\
u(0, x)=u_{0}(x), \text { for } x \in \mathbb{R}^{n} .
\end{array}\right.
$$

Note that $\nu(x)$ corresponds to the effect of the environment on the species local growth rate; this model has been investigated for $n=1$ in (Hamel et al., 2010). In this case, the negative effect of the fragmentation of $u_{0}$ may be compensated by the fact that fragmented supports may have more chances to intersect with favorable regions. Thus, we could expect nontrivial intertwined effects between the level of environmental heterogeneity and the fragmentation of the initial condition. Other reaction-diffusion based models enable evolution and adaption (Kirkpatrick and Barton, 1997; Pease et al., 1989). In a recent study, Kanarek and Webb (2010) have shown that, for species which can adaptively respond to overcome Allee effects, even small invading populations can survive. In such cases, fragmentation may also have a different effect.

\section{A Proofs of Theorem 2 and Lemma 2}

Proof of Theorem 2. For all $\alpha \geq 0$ and $L>0$, we denote the solution of (2.1) with the initial condition (2.4) by $u^{L, \alpha}(t, x)$.

1. We first show that $L^{*}(\alpha) \leq 2 L^{*}(0)$ for all $\alpha \geq 0$ and that $L^{*}(\alpha) \rightarrow 2 L^{*}(0)$ as $\alpha \rightarrow \infty$.

Fix $\alpha \geq 0$. Let $\varepsilon \in\left(0,2 L^{*}(0)\right)$, and set $L^{\varepsilon}=L^{*}(0)-\varepsilon / 2$. Let $v(t, x)=u^{L^{\varepsilon}, 0}\left(t, x-L^{\varepsilon} / 2-\alpha / 2\right)$ for all $t \geq 0$ and $x \in \mathbb{R}$. From Theorem $1(\mathrm{i}), u^{L^{\varepsilon}, 0}(t, x) \rightarrow 0$ uniformly in $\mathbb{R}$ as $t \rightarrow \infty$. Thus, we obtain the existence of some $t^{*}>0$ such that

$$
0 \leq v(t, x)=u^{L^{\varepsilon}, 0}\left(t, x-L^{\varepsilon} / 2-\alpha / 2\right)<\rho / 4 \text { for all } t \geq t^{*} \text { and } x \in \mathbb{R} .
$$

Now, let $w:=u^{2 L^{\varepsilon}, \alpha}-v$. Then $w(0, \cdot)=\chi_{\left[-\left(\alpha / 2+L^{\varepsilon}\right),-\alpha / 2\right]}$. Since $0 \leq u(0, \cdot), v(0, \cdot), w(0, \cdot) \leq 1$ and $f(0)=f(1)=0$, it follows from the parabolic maximum principle (Ch. 2 in Friedman (1964) and Ch. 3 in Protter and Weinberger (1967)) that $u, v, w \in[0,1]$. Moreover, $w$ satisfies:

$$
w_{t}=D w_{x x}+f\left(u^{2 L^{\varepsilon}, \alpha}\right)-f(v) \leq D w_{x x}+K w,
$$

with $K=\left\|f^{\prime}\right\|_{L^{\infty}(0,1)}$. 
Let $\phi$ be the solution to the linear equation $\phi_{t}=D \phi_{x x}+K \phi$ with the initial condition $\phi(0, x)=w(0, x)$. Then $\phi\left(t^{*}, 0\right) \rightarrow 0$ as $\alpha \rightarrow \infty$. Thus, for adequately large $\alpha, \phi\left(t^{*}, 0\right)<\rho / 4$. Since $x \mapsto \phi\left(t^{*}, x\right)$ is decreasing in $[0, \infty)$, we obtain:

$$
\phi\left(t^{*}, x\right)<\rho / 4 \text { for all } x \geq 0 \text { and } \alpha \text { large enough. }
$$

Moreover, the maximum principle implies that $w \leq \phi$ in $[0, \infty) \times \mathbb{R}$. Then $u^{2 L^{\varepsilon}, \alpha}=v+w \leq \phi+v$ in $[0, \infty) \times \mathbb{R}$, and finally, using (A.14) and (A.16), we obtain

$$
u^{2 L^{\varepsilon}, \alpha}\left(t^{*}, x\right)<\rho / 2 \text { for all } x \geq 0 \text { and } \alpha>0 \text { large enough. }
$$

Therefore, the evenness of $u^{2 L^{\varepsilon}, \alpha}$ implies that $u^{2 L^{\varepsilon}, \alpha}\left(t^{*}, x\right)<\rho / 2$ for all $x \in \mathbb{R}$ and $\alpha>0$ large enough. Comparing with the solution of the ODE $N^{\prime}=f(N)$ with $N\left(t^{*}\right)=\rho / 2$, for $\alpha$ large enough, we obtain that $u^{2 L^{\varepsilon}, \alpha}(t, x) \rightarrow 0$ uniformly in $x \in \mathbb{R}$ as $t \rightarrow \infty$. According to the definition of $L^{*}(\alpha)$, this implies that $L^{*}(\alpha)>2 L^{\varepsilon}$, i.e.,

$$
2 L^{*}(0)-\varepsilon<L^{*}(\alpha) \text { for } \alpha>0 \text { large enough. }
$$

Since $\varepsilon>0$ can be arbitrarily small, we conclude that

$$
\liminf _{\alpha \rightarrow+\infty} L^{*}(\alpha) \geq 2 L^{*}(0) .
$$

Let us now check that for all $\alpha \geq 0, L^{*}(\alpha)<2 L^{*}(0)$. Let $\alpha \geq 0$ be fixed. For all $L>0$, we have $u^{L, \alpha}(0, \cdot) \geq u^{L / 2,0}(0, \cdot-\alpha / 2-L / 4)$. Thus, the parabolic maximum principle leads to

$$
u^{L, \alpha}(t, \cdot) \geq u^{L / 2,0}(t, \cdot-\alpha / 2-L / 4) \text { for all } t \geq 0 .
$$

Take $L=2 L^{*}(0)$. Theorem 1 (iii) shows that $u^{L^{*}(0), 0}\left(t, \cdot-\alpha / 2-L^{*}(0) / 2\right)$ converges uniformly in $\mathbb{R}$ as $t \rightarrow+\infty$ to the stationary solution $\tilde{u}\left(\cdot-\alpha / 2-L^{*}(0) / 2\right)$ of $(2.1)$, where $\tilde{u}$ verifies $\tilde{u}(0)=\rho_{1}>\rho>0$, $\tilde{u}^{\prime}(0)=0$ and $\tilde{u}$ is symmetrically decreasing. Thus, using (A.18), we get

$$
u^{2 L^{*}(0), \alpha} \nrightarrow 0 \text { as } t \rightarrow+\infty .
$$

Theorem 1 (i) thus implies that $L^{*}(\alpha) \leq 2 L^{*}(0)$. Assume that $L^{*}(\alpha)=2 L^{*}(0)$. Then, from Theorem 1 (iii), we know that $u^{2 L^{*}(0), \alpha}$ converges to $\tilde{u}$, uniformly in $\mathbb{R}$ as $t \rightarrow+\infty$. Using inequality (A.18), we obtain

$$
\tilde{u}(x) \geq \tilde{u}\left(x-\alpha / 2-L^{*}(0) / 2\right) \text { for all } x \in \mathbb{R} .
$$

In particular, we get $\tilde{u}\left(\alpha / 2+L^{*}(0) / 2\right) \geq \tilde{u}(0)$ which is impossible since $\tilde{u}$ is symmetrically decreasing. Thus, we finally obtain

$$
L^{*}(\alpha)<2 L^{*}(0)
$$

and together with (A.17), this shows that $L^{*}(\alpha) \rightarrow 2 L^{*}(0)$ as $\alpha \rightarrow+\infty$. 
2. We prove that the function $\alpha \mapsto L^{*}(\alpha)$ is continuous on $[0, \infty)$. For any $0 \leq \alpha \leq \beta$, we have $u^{L^{*}(\alpha)-(\beta-\alpha), \beta}(0, \cdot) \leq u^{L^{*}(\alpha), \alpha}(0, \cdot)$ if $L^{*}(\alpha)-(\beta-\alpha)>0$. Therefore, the parabolic maximum principle together with Theorem 1 imply that

$$
L^{*}(\beta) \geq L^{*}(\alpha)-(\beta-\alpha), \text { for all } 0 \leq \alpha \leq \beta,
$$

whenever $L^{*}(\alpha)-(\beta-\alpha)$ is positive or not. In other words, the function $\alpha \mapsto L^{*}(\alpha)+\alpha$ is non-decreasing, which implies that the function $\alpha \mapsto L^{*}(\alpha)$ is right lower and left upper semicontinuous. In order to complete the proof of Theorem 2, one is left to prove that the function $\alpha \mapsto L^{*}(\alpha)$ is right upper and left lower semicontinuous.

Step (i). We first show that the function $\alpha \mapsto L^{*}(\alpha)$ is right upper semicontinuous on $[0,+\infty)$. Let


Assume now that $\overline{L^{+}}>L^{*}(\alpha)$ and set

$$
\tilde{L}=\frac{\overline{L^{+}}+L^{*}(\alpha)}{2} .
$$

From the definition of $\overline{L^{+}}$we know that there exists a decreasing sequence $\left(\alpha_{n}\right)_{n \geq 0}$ such that $\alpha_{n} \in(\alpha, \alpha+\tilde{L})$ for all $n \geq 0, \alpha_{n} \rightarrow \alpha$ and $L^{*}\left(\alpha_{n}\right) \rightarrow \overline{L^{+}}$as $n \rightarrow \infty$. Since $\overline{L^{+}}>\tilde{L}$, there exists $N \geq 0$ such that for all $n \geq N, L^{*}\left(\alpha_{n}\right)>\tilde{L}$. Then, from Theorem 1 Part (i), for all $n \geq N$, we have

$$
u^{\tilde{L}, \alpha_{n}}(t, \cdot) \rightarrow 0 \text { uniformly in } \mathbb{R} \text { as } t \rightarrow \infty,
$$

which in turn implies that

$$
u^{\tilde{L}-\left(\alpha_{n}-\alpha\right), \alpha_{n}}(t, \cdot) \rightarrow 0 \text { uniformly in } \mathbb{R} \text { as } t \rightarrow \infty
$$

since $0<\tilde{L}-\left(\alpha_{n}-\alpha\right)<\tilde{L}$. Then, define the family $\left(\phi_{\lambda}\right)_{\lambda \geq 0}$ as follows

$$
\phi_{\lambda}(\cdot)= \begin{cases}u^{\lambda, \tilde{L}+\alpha-\lambda}(0, \cdot), & \text { for } \lambda \in[0, \tilde{L}), \\ u^{\lambda, \alpha}(0, \cdot), & \text { for } \lambda \geq \tilde{L} .\end{cases}
$$

This is a monotone increasing family in the sense of Theorem 1.3 in (Du and Matano, 2010). Since $\tilde{L}>$ $L^{*}(\alpha)$, it follows from Theorem 1 Part (ii) that the solution $u^{\tilde{L}, \alpha}$ of $(2.1)$ with initial condition $\phi_{\tilde{L}}$ verifies $u^{\tilde{L}, \alpha} \rightarrow 1$ uniformly on compacts as $t \rightarrow \infty$. Besides, we know from (A.22) that the solution $u^{\tilde{L}-\left(\alpha_{N}-\alpha\right), \alpha_{N}}$ of (2.1) with initial condition $\phi_{\tilde{L}-\left(\alpha_{N}-\alpha\right)}$ converges to 0 uniformly in $\mathbb{R}$ as $t \rightarrow \infty$. Since $\tilde{L}-\left(\alpha_{N}-\alpha\right)<\tilde{L}$, it follows then from Theorem 1.3 in (Du and Matano, 2010) that there exists $\tilde{\lambda} \in\left(\tilde{L}-\left(\alpha_{N}-\alpha\right), \tilde{L}\right)$ such that for all $\lambda>\tilde{\lambda}$, the solution of (2.1) with initial condition $\phi_{\lambda}$ converges to 1 uniformly on compacts as $t \rightarrow \infty$. Thus, for any $N_{1}>N$ such that $\tilde{L}-\left(\alpha_{N_{1}}-\alpha\right)>\tilde{\lambda}$, the solution of (2.1) with initial condition $\phi_{\tilde{L}-\left(\alpha_{N_{1}}-\alpha\right)}$ converges to 1 uniformly on compacts as $t \rightarrow \infty$. This means that $u^{\tilde{L}-\left(\alpha_{N_{1}}-\alpha\right), \alpha_{N_{1}}} \rightarrow 1$ uniformly on compacts as $t \rightarrow \infty$ and contradicts (A.22). Thus, we have shown that $\overline{L^{+}}=L^{*}(\alpha)$. This means that the function $\alpha \mapsto L^{*}(\alpha)$ is right upper semicontinuous on $[0,+\infty)$. As it is already known to be right lower semicontinuous, it is then right-continuous. 
Step (ii). We show that the function $\alpha \mapsto L^{*}(\alpha)$ is left lower semicontinuous in $(0,+\infty)$. Let $\alpha>0$, and set $\underline{L^{-}}=\lim \inf _{\beta \rightarrow \alpha^{-}} L^{*}(\beta)$. The inequality (A.20) implies that $\underline{L^{-}} \in\left[0, L^{*}(\alpha)\right]$. Assume by contradiction that $\underline{L^{-}}<L^{*}(\alpha)$ and set

$$
\hat{L}=\frac{L^{-}+L^{*}(\alpha)}{2} .
$$

Let $\left(\alpha_{n}\right)_{n \geq 0}$ be a positive and increasing sequence such that $\alpha_{n} \rightarrow \alpha$ and $L^{*}\left(\alpha_{n}\right) \rightarrow \underline{L^{-}}$as $n \rightarrow \infty$. Since $\underline{L^{-}}<\hat{L}$, there exists $N \geq 0$ such that for all $n \geq N, L^{*}\left(\alpha_{n}\right)<\hat{L}$ and therefore, from Theorem 1 Part (ii), $u^{\hat{L}, \alpha_{n}}(t, \cdot) \rightarrow 1$ uniformly on compacts as $t \rightarrow \infty$. As a consequence, we also have, for all $n \geq N$,

$$
u^{\hat{L}+\alpha-\alpha_{n}, \alpha_{n}}(t, \cdot) \rightarrow 1 \text { uniformly on compacts as } t \rightarrow \infty,
$$

since $0<\hat{L}<\hat{L}+\alpha-\alpha_{n}$. Let us define the family $\left(\psi_{\lambda}\right)_{\lambda \geq 0}$ by

$$
\psi_{\lambda}(\cdot)= \begin{cases}u^{\lambda, \hat{L}+\alpha-\lambda}(0, \cdot), & \text { for } \lambda \in[0, \hat{L}+\alpha), \\ u^{\lambda, 0}(0, \cdot), & \text { for } \lambda \geq \hat{L}+\alpha\end{cases}
$$

The family $\left(\psi_{\lambda}\right)_{\lambda \geq 0}$ is monotone increasing. Furthermore, since $\hat{L}<L^{*}(\alpha)$, the solution $u^{\hat{L}, \alpha}$ of $(2.1)$ with initial condition $\psi_{\hat{L}}$ converges to 0 uniformly in $\mathbb{R}$ as $t \rightarrow \infty$. Conversely, we know (see eq. (A.23)) that the solution $u^{\hat{L}+\alpha-\alpha_{N}, \alpha_{N}}$ of (2.1) with initial condition $\psi_{\hat{L}+\alpha-\alpha_{N}}$ converges to 1 uniformly on compacts as $t \rightarrow \infty$. Thus, using again the result of Theorem 1.3 in (Du and Matano, 2010), we obtain the existence of $\hat{\lambda} \in\left(\hat{L}, \hat{L}+\alpha-\alpha_{N}\right)$ such that, for all $\lambda<\hat{\lambda}$, the solution of (2.1) with initial condition $\psi_{\lambda}$ converges to 0 as $t \rightarrow \infty$. Let $N_{1} \geq N$ be such that $\hat{L}+\alpha-\alpha_{N_{1}}<\hat{\lambda}$. The above result implies that the solution of (2.1) with initial condition $\psi_{\hat{L}+\alpha-\alpha_{N_{1}}}$ converges to 0 as $t \rightarrow \infty$. This contradicts (A.23). Thus, we have proved that $\underline{L^{-}}=L^{*}(\alpha)$. This means that the function $\alpha \mapsto L^{*}(\alpha)$ is left lower semicontinuous on $(0,+\infty)$. As it is already known to be left upper semicontinuous, it is then left-continuous.

Finally, we have shown that the function $\alpha \mapsto L^{*}(\alpha)$ is continuous on $[0,+\infty)$ and the proof of Theorem 2 is thereby complete.

Proof of Lemma 2. For any $\alpha \geq 0, L>0$ and $\rho \in(0,1 / 2)$, we denote the solution of (2.1), (2.4) by $u_{\rho}^{L, \alpha}(t, x)$, where $f$ is defined by (3.11). Since $f(0)=f(1)=0$ and $u_{\rho}^{L, \alpha}(0, x) \in[0,1]$, it follows from the parabolic maximum principle that $u_{\rho}^{L, \alpha}(t, x) \in[0,1]$ for all $t \geq 0$ and $x \in \mathbb{R}$.

Let $0<\rho_{1} \leq \rho_{2}<1 / 2$. Then, for all $s \in[0,1], s(1-s)\left(s-\rho_{1}\right) \geq s(1-s)\left(s-\rho_{2}\right)$. Since $u_{\rho_{2}}^{L, \alpha}(t, x) \in[0,1]$ for all $t \geq 0$ and $x \in \mathbb{R}, u_{\rho_{2}}^{L, \alpha}$ is a subsolution of the equation satisfied by $u_{\rho_{1}}^{L, \alpha}$. It follows from the parabolic maximum principle that $u_{\rho_{1}}^{L, \alpha} \geq u_{\rho_{2}}^{L, \alpha}$.

\section{B Numerical solution of (2.1)}

The equation (with $n=1$ and $n=2$ ) was solved using Comsol Multiphysics ${ }^{\circledR}$ time-dependent solver, using second order finite element method (FEM). This solver uses a so-called lines approach method 
incorporating variable-order variable-stepsize backward differentiation formulas. Nonlinearity are treated using a Newton's method.

In the 1D case, for the numerical computation of $L^{*}(\alpha)$, we used a so-called "large" time $(t=50)$. The solution of (2.1) was estimated by solving the problem on the bounded interval $\Omega=(-100,100)$ with Dirichlet boundary conditions. In the 2D case, we estimated the solution of (2.1) by solving the problem on a bounded domain $\Omega=(-100,100) \times(-100,100)$ with Dirichlet boundary conditions.

In both $1 \mathrm{D}$ and $2 \mathrm{D}$ cases, the outcome of the invasion was measured at $t=50$ : the establishment stage was considered to be successful if and only if $\min _{x \in \Omega} u(50, x)>\rho$, where $\rho$ is the Allee threshold defined in (2.3). Note that this definition can lead to overestimate the success rate of the establishment stage if the time required for the population density to be uniformly below $\rho$ is larger than 50 . However, in most cases except when $\rho$ was very close to $1 / 2$, we observed that the outcome of the establishment stage was already clear at $t=50$.

\section{Acknowledgements}

The authors are supported by the French "Agence Nationale de la Recherche" within the projects ColonSGS, PREFERED and URTICLIM. The third author is also indebted to the Alexander von Humboldt Foundation for its support.

\section{References}

Allee, W. C. (1938). The social life of animals. Norton, New York.

Aronson, D. G. and H. G. Weinberger (1978). Multidimensional Non-Linear Diffusion Arising in PopulationGenetics. Advances in Mathematics 30(1), 33-76.

Berec, L., E. Angulo, and F. Courchamp (2007). Multiple Allee effects and population management. Trends in Ecology 83 Evolution 22, 185-191.

Berestycki, H., F. Hamel, and G. Nadin (2008). Asymptotic spreading in heterogeneous diffusive excitable media. Journal of Functional Analysis 255(9), 2146-2189.

Berestycki, H., F. Hamel, and L. Roques (2005). Analysis of the periodically fragmented environment model: I - Species persistence. Journal of Mathematical Biology 51(1), 75-113.

Berestycki, H., F. Hamel, and L. Rossi (2007, Jul). Liouville-Type Results For Semilinear Elliptic Equations in Unbounded Domains. Annali Di Matematica Pura Ed Applicata 186(3), 469-507.

Cantrell, R, S. and C. Cosner (2003). Spatial ecology via reaction-diffusion equations. John Wiley \& Sons Ltd, Chichester, UK . 
DAISIE (2009). Handbook of alien species in Europe. Springer, Dordrecht.

DAISIE (2010). Alien terrestrial arthropods of Europe. BioRisk 4, Volume 1 and 2. Pensoft, Sofia-Moscow.

Dennis, B. (1989). Allee effects: population growth, critical density, and the chance of extinction. Natural Resource Modeling 3, 481-538.

Dobson, A. P. and R. M. May (1986). Patterns of invasions by pathogens and parasites. In H. A. Mooney and J. A. Drake (Eds.), Ecology of Biological Invasions of North America and Hawaii, pp. 58-76. Springer Verlag, New York.

Drake, J. M. (2004). Allee effects and the risk of biological invasion. Risk Analysis 24, 795-802.

Drury, K. L. S., J. M. Drake, D. M. Lodge, and G. Dwyer (2007). Immigration events dispersed in space and time: Factors affecting invasion success. Ecological Modelling 206, 63-78.

Du, Y. and H. Matano (2010). Convergence and sharp thresholds for propagation in nonlinear diffusion problems. Journal of the European Mathematical Society, to appear.

Fahrig, L. (2003). Effects of habitat fragmentation on biodiversity. Annual Review of Ecology, Evolution, and Systematics 34, 487-515.

Fife, P. C. (1979). Long-time behavior of solutions of bistable non-linear diffusion equations. Archive for Rational Mechanics and Analysis 70(1), 31-46.

Fife, P. C. and J. McLeod (1977). The approach of solutions of nonlinear diffusion equations to traveling front solutions. Archive for Rational Mechanics and Analysis 65(1), 335-361.

Fisher, R. A. (1937). The wave of advance of advantageous genes. Annals of Eugenics 7, 335-369.

Friedman, A. (1964). Partial differential equations of parabolic type. Prentice-Hall, Englewood Cliffs, NJ.

Gardner, R. H., B. T. Milne, M. G. Turner, and R. V. O'Neill (1987). Neutral models for the analysis of broad-scale landscape pattern. Landscape Ecology 1, 19-28.

Hamel, F., J. Fayard, and L. Roques (2010). Spreading speeds in slowly oscillating environments. Bulletin of Mathematical Biology 72(5), 1166-1191.

Harary, F. and H. Harborth (1976). Extremal animals. Journal of Combinatorics, Information 83 System Sciences 1, 1-8.

IUCN (2000). Guidelines for the prevention of biodiversity loss caused by alien invasive species prepared by the Species Survival Commission (SSC) invasive species specialist group. Approved by the 51st Meeting of the IUCN Council, Gland. 
IUCN (2002). Policy recommendations papers for sixth meeting of the Conference of the Parties to the Convention on Biological Diversity (COP6). The Hague, Netherlands.

Kanarek, A. R. and C. T. Webb (2010). Allee effects, adaptive evolution, and invasion success. Evolutionary Applications 3, 122-135.

Kanel, J. I. (1964). Stabilization of solutions of the equations of combustion theory with finite initial functions. Matematicheskii Sbornik 65, 398-413.

Keitt, T. H. (2000). Spectral representation of neutral landscapes. Landscape Ecology 15, 479-494.

Keitt, T. H., M. A. Lewis, and R. D. Holt (2001). Allee effects, invasion pinning, and species' borders. American Naturalist 157, 203-216.

Kenis, M. (2006). Insects-insecta. In R. Wittenberg (Ed.), Invasive Alien Species in Switzerland. An inventory of alien species and their threat to biodiversity and economy in Switzerland, pp. 131211. Swiss Confederation - Federal Office for the Environment Environmental Studies.

Kenis, M., M.-A. Auger-Rozenberg, A. Roques, L. Timms, C. Péré, M. J. W. Cock, J. Settele, S. Augustin, and C. Lopez-Vaamonde (2009). Ecological effects of invasive alien insects. Biological Invasions 11(1), $21-45$.

Kirkpatrick, M. and N. H. Barton (1997). Evolution of a species'range. The American Naturalist 150, $1-23$.

Kolmogorov, A. N., I. G. Petrovsky, and N. S. Piskunov (1937). Étude de l'équation de la diffusion avec croissance de la quantité de matière et son application à un problème biologique. Bulletin de l'Université d'État de Moscou, Série Internationale A 1, 1-26.

Kramer, A. M., B. Dennis, A. M. Liebhold, and J. M. Drake (2009). The evidence for Allee effects. Population Ecology 51, 341-354.

Lande, R. (1998). Demographic stochasticity and Allee effect on a scale with isotrophic noise. Oikos 83, $353-358$.

Leung, B., J. M. Drake, and D. M. Lodge (2004). Predicting invasions: propagule pressure and the gravity of Allee effects. Ecology 85, 1651-1660.

Lewis, M. A. and P. Kareiva (1993). Allee dynamics and the speed of invading organisms. Theoretical Population Biology 43, 141-158.

Mccarthy, M. A. (1997). The Allee effect, finding mates and theoretical models. Ecological Modelling 103(1), 99-102. 
Murray, J. D. (2002). Mathematical Biology. Third Edition. Interdisciplinary Applied Mathematics 17, Springer-Verlag, New York.

Pease, C. P., R. Lande, and J. J. Bull (1989). A model of population growth, dispersal and evolution in a changing environment. Ecology 70, 1657-1664.

Protter, M. H. and H. F. Weinberger (1967). Maximum Principles in Differential Equations. Prentice-Hall, Englewood Cliffs, NJ.

Richardson, D. M., P. Py̌sek, M. Rejmánek, M. G. Barbour, F. Dane Panetta, and C. J. West (2000). Naturalization and invasion of alien plants: concepts and definitions. Diversity and Distributions 6, 93-107.

Roques, A., W. Rabitsch, J.-Y. Rasplus, C. Lopez-Vaamonde, W. Nentwig, and M. Kenis (2009). Alien terrestrial invertebrates of Europe. Dordrecht, Netherlands: Springer.

Roques, L. and M. D. Chekroun (2007). On population resilience to external perturbations. SIAM Journal on Applied Mathematics 68(1), 133-153.

Roques, L. and M. D. Chekroun (2010). Does reaction-diffusion support the duality of fragmentation effect? Ecological Complexity 7, 100-106.

Roques, L. and F. Hamel (2007). Mathematical analysis of the optimal habitat configurations for species persistence. Mathematical Biosciences 210(1), 34-59.

Roques, L., A. Roques, H. Berestycki, and A. Kretzschmar (2008). A population facing climate change: joint influences of Allee effects and environmental boundary geometry. Population Ecology 50(2), 215225 .

Roques, L. and R. S. Stoica (2007). Species persistence decreases with habitat fragmentation: an analysis in periodic stochastic environments. Journal of Mathematical Biology 55(2), 189-205.

Shigesada, N. and K. Kawasaki (1997). Biological invasions: theory and practice. Oxford Series in Ecology and Evolution, Oxford: Oxford University Press.

Skellam, J. G. (1951). Random dispersal in theoretical populations. Biometrika 38, 196-218.

Turchin, P. (1998). Quantitative analysis of movement: measuring and modeling population redistribution in animals and plants. Sinauer Associates, Sunderland, MA.

Veit, R. R. and M. A. Lewis (1996). Dispersal, population growth, and the Allee effect: dynamics of the house finch invasion of eastern North America. American Naturalist 148, 255-274. 
Vilà, M., B. C, P. Pyšek, M. Josefsson, P. Genovesi, S. Gollasch, W. Nentwig, S. Olenin, A. Roques, D. Roy, P. E. Hulme, and D. partners (2009). How well do we understand the impacts of alien species on ecosystem services? A pan-European cross-taxa assessment. Frontiers in Ecology and the Environment.

Walther, G.-R., A. Roques, P. E. Hulme, M. T. Sykes, P. Pyšek, I. Kühn, M. Zobel, S. Bacher, Z. BottaDukát, H. Bugmann, B. Czúcz, J. Dauber, T. Hickler, V. Jarošík, M. Kenis, S. Klotz, D. Minchin, M. Moora, W. Nentwig, J. Ott, V. E. Panov, B. Reineking, C. Robinet, V. Semenchenko, W. Solarz, W. Thuiller, M. Vilà, K. Vohland, and J. Settele (2009). Alien species in a warmer world: risks and opportunities. Trends in Ecology \& Evolution 24 (12), 686-693.

Yamanaka, T. and A. M. Liebhold (2009). Mate-location failure, the Allee effect, and the establishment of invading populations. Population Ecology 51, 337-340.

Zlatoš, A. (2006). Sharp transition between extinction and propagation of reaction. Journal of the American Mathematical Society 19, 251-263. 